\title{
Asprosin, a C-Terminal Cleavage Product of Fibrillin 1 Encoded by the FBN1 Gene, in Health and Disease
}

\author{
Mehmet Akif Ovalia ${ }^{a}$ lbrahim Bozgeyik ${ }^{b}$ \\ aDepartment of Physiology, Faculty of Medicine, Çanakkale Onsekiz Mart University, Çanakkale, Turkey; \\ ${ }^{b}$ Department of Medical Biology, Faculty of Medicine, Adiyaman University, Adiyaman, Turkey
}

\section{Keywords}

Asprosin · FBN1 - Marfan syndrome · Neonatal progeroid syndrome $\cdot$ Fibrillin 1

\begin{abstract}
Background: Asprosin is a novel fasting-induced, glucogenic, and orexigenic protein hormone that is discovered with the help of genetic studies in patients with neonatal progeroid syndrome. Asprosin is encoded by the penultimate 2 exons (65 and 66) of the fibrillin 1 (FBN1) gene. Profibrillin 1 is the unprocessed protein product of FBN1 and undergoes a proteolytic cleavage by furin enzyme to produce mature fibrillin 1 and asprosin. The main organ responsible for the asprosin production seems to be white adipose tissue. Summary: Asprosin promotes hepatic glucose release in the liver and appetite stimulation in the hypothalamus through activation of the CAMP signaling circuitry through interacting with its $G$ protein-coupled receptor, called OR4M1. Increasing mass of evidence suggests that asprosin is involved in the development and progression of various clinical conditions including diabetes, obesity, cardiomyopathy, cancer, and polycystic ovarian syndrome. It regulates various cellular and physiological processes such as appetite stimulation, glucose release, insulin secretion, apoptotic cell death, and inflammatory response. In this review, we discuss
\end{abstract}

the current literature on asprosin and try to shed light on the yet undiscovered functions of asprosin. Key Message: Asprosin is a key regulatory factor for preserving the homeostasis of energy metabolism.

(c) 2022 S. Karger AG, Basel

\section{Introduction}

Asprosin is a fasting-responsive orexigenic protein hormone which enhances hepatic glucose release in the liver and hypothalamic appetite stimulation through the G protein-coupled receptor-dependent cAMP signaling pathway [Duerrschmid et al., 2017]. Asprosin, which is a C-terminal cleavage product of profibrillin, was first identified by Romere et al. [2016] in patients diagnosed with neonatal progeroid syndrome that harbor similar $3^{\prime}$ truncating mutations in the fibrillin 1 (FBN1) gene as characterized by Sanger and whole-exome sequencing analysis. As presented in Figure 1, asprosin is mainly produced by white adipose tissue and circulates in nanomolar levels in the blood. It is taken up by the liver where it stimulates the G protein-cAMP-PKA axis, leading to quick release of glucose into the circulation [Romere et al., 2016].

Correspondence to:

Ibrahim Bozgeyik, i.bozgeyik@gmail.com

karger@karger.com

(C) 2022 S. Karger AG, Basel

Karger ${ }^{\prime \prime}=$ 


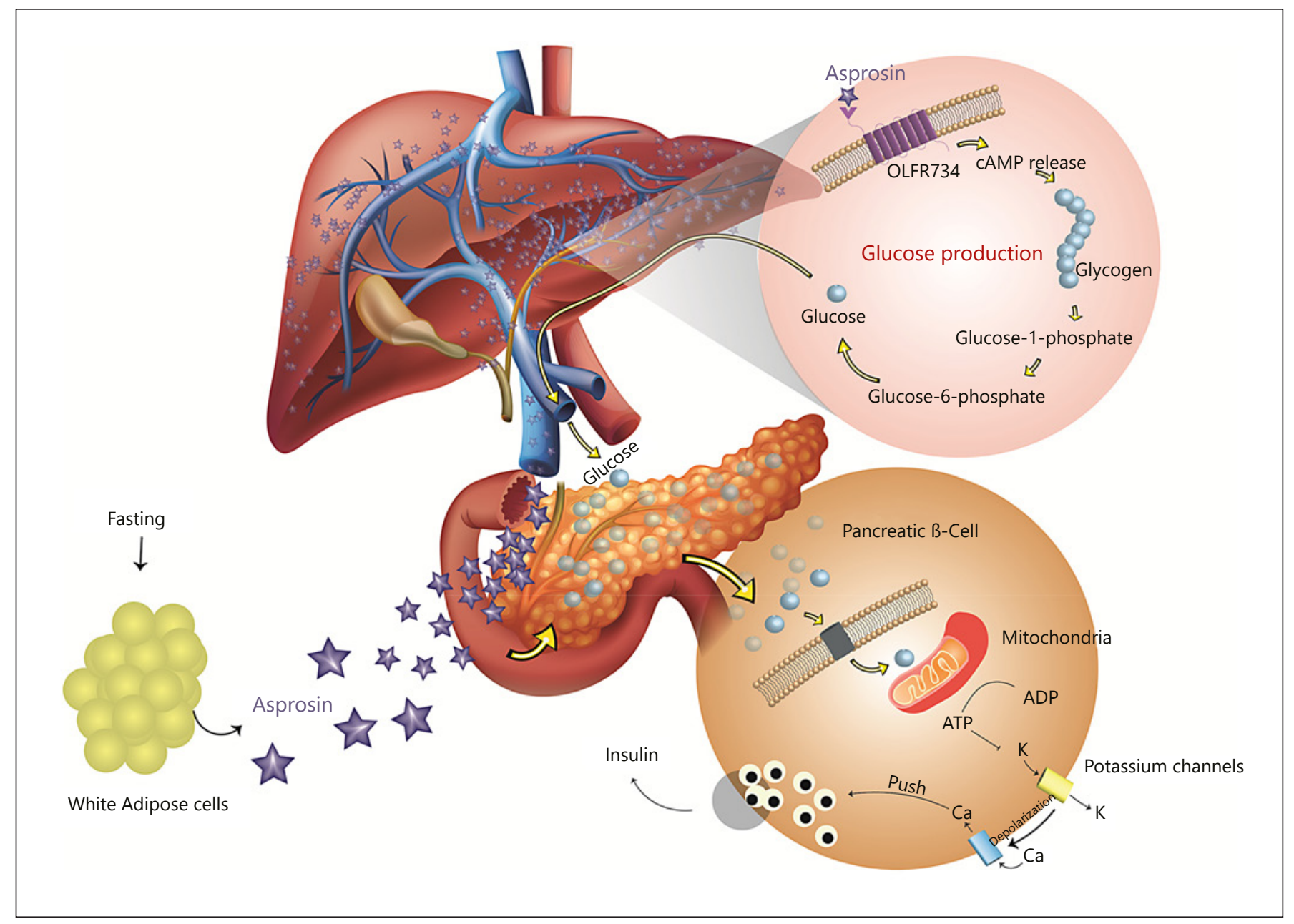

Fig. 1. Asprosin responds to low glucose concentration and is secreted mainly from white adipose tissues in response to fasting conditions. Once secreted into the bloodstream, asprosin travels to the liver where it activates production and release of glucose into the circulation by interacting with its receptor OR4M1 (human ortholog of OLFR734) and activates the G protein-cAMP-PKA signaling circuitry. Asprosin can also exit the circulation and cross the blood-brain barrier to regulate appetite stimulation through inducing orexigenic AgRP neurons and suppressing anorexigenic POMC neurons (not shown in figure).
Asprosin promotes gluconeogenesis and maintains glucose homeostasis via its $G$ protein-coupled receptor, olfactory receptor family 4 subfamily $M$ member 1 (OR4M1) [Li et al., 2019]. OR4M1 is the human ortholog of olfactory receptor 734 (OLFR734) in mice. Olfr 734 knockout mice were associated with the blunted response to asprosin and reduced glucose production in response to both high-fat diet and fasting [Li et al., 2019]. Upon binding to its receptor OLFR734, asprosin activates the cAMP signaling pathway to enhance hepatic glucose release and maintains glucose homeostasis during fasting and in obesity [Li et al., 2019]. Olfactory receptor family of proteins are mainly involved in detection of chemical stimuli that contribute to olfactory perception. However, whether OLFR734 responds to odorant molecules and how these molecules and asprosin regulate olfactory perception and food intake as well as energy homeostasis still remains mysterious.

The concentration of asprosin in the circulation increases with hunger and decreases acutely with refeeding, showing circadian rhythm in coordination with nutritional status [Romere et al., 2016]. Humans and mice with insulin resistance have pathologically high plasma asprosin levels [Romere et al., 2016]. The loss of function of asprosin by immunological or genetic approaches has a strong glucose- and insulin-lowering effect due to de- 


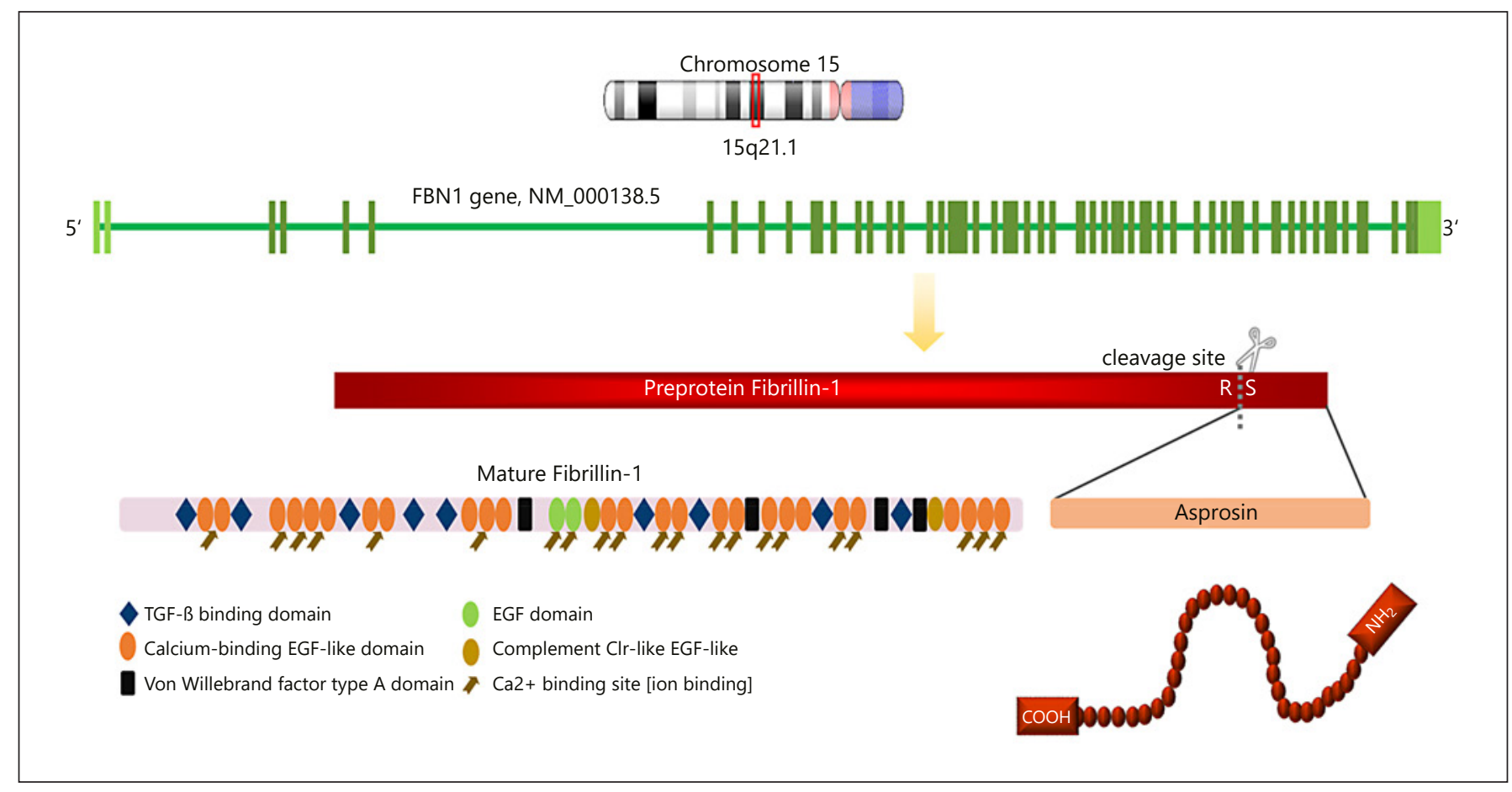

Fig. 2. The gene responsible for asprosin production is fibrillin 1 (FBN1), which is located in chromosome 15q21.1. This gene encodes a member of the fibrillin family of proteins. The encoded preproprotein, called profibrillin-1 (2,871 amino acids), undergoes proteolytic cleavage to generate 2 separate proteins - the ex- tracellular matrix component fibrillin 1 and the protein hormone asprosin. The FBN1 gene consists of 66 exons and the penultimate 2 exons (exons 65 and 66) of FBN1 are responsible for the asprosin production. Asprosin is 140 amino acids in length and approximately $30 \mathrm{kDa}$ in molecular weight. creased hepatic glucose release, strongly indicating that asprosin is pathologically related with the insulin resistance of human and mouse [Romere et al., 2016]. Reduction of asprosin prevents hyperinsulinization due to metabolic syndrome [Mishra et al., 2021]. Moreover, peripheral intravenous administration of asprosin was shown to cross the blood-brain barrier, where it directly triggers orexigenic AgRP+ neurons in a cAMP-dependent manner for the activation of the hypothalamic feeding circuitry and stimulation of appetite [Duerrschmid et al., 2017].

\section{Genetics}

Asprosin is a C-terminal cleavage product of fibrillin 1, which is encoded by the FBN1 gene located at the 15q21.1 chromosomal region (Fig. 2) [Romere et al., 2016]. FBN1 is composed of 66 exons, and the last 2 exons (65 and 66) were identified to be responsible for asprosin production [Romere et al., 2016]. Fibrillin 1 is synthesized as a preproprotein which undergoes proteolytic cleavage for the generation of 2 separate proteins - a structural extracellular matrix glycoprotein, fibrillin 1 , and a secreted protein hormone, asprosin [Romere et al., 2016]. The unprocessed fibrillin 1 preprotein is 2,871 amino acids in length [Romere et al., 2016]. Proteolytical cleavage of this preprotein from its $\mathrm{C}$-terminal end by the activity of protease furin yields 2,704-amino-acid-long mature fibrillin 1 protein and 140-amino-acid-long asprosin [Romere et al., 2016]. Compared to its recombinantly expressed bacterial counterpart which has a molecular weight of approximately $17 \mathrm{kDa}$, human asprosin runs at $\sim 30 \mathrm{kDa}$ in SDSPAGE, indicating that asprosin undergoes post-translational modifications (predicted to have $3 \mathrm{~N}$-linked glycosylation sites) [Romere et al., 2016]. Immunoblotting of recombinantly expressed asprosin protein in mammalian cells revealed an approximately $30-\mathrm{kDa}$ protein which likely clarifies the difference in molecular weight between mammalian and recombinant bacterial asprosin [Romere et al., 2016]. Mutations in the FBN1 gene are significantly associated with the neonatal progeroid syndrome (NPS) [Graul-Neumann et al., 2010; 
Goldblatt et al., 2011; Horn and Robinson, 2011; Takenouchi et al., 2013; Jacquinet et al., 2014; Romere et al., 2016], Marfan syndrome [Robinson et al., 2002], MASS phenotype, marfanoid habitus, and familial ectopia lentis [Brautbar et al., 2010]. 3' truncating mutations in FBN1 were linked to the genetic ablation of asprosin in patients with NPS [Romere et al., 2016]. Patients with NPS are generally characterized by partial lipodystrophy and progeroid appearance, and display extreme leanness and hypophagia that is associated with deficient plasma asprosin levels [O'Neill et al., 2007]. Seven patients with this syndrome have been reported and all have $3^{\prime}$ truncating mutations in the FBN1 gene, and these mutations have been shown to accumulate in the penultimate exon of FBN1 and were predicted to escape nonsense-mediated decay, which eventually leads to the production of a truncated, mutant profibrillin protein [Romere et al., 2016]. Expression of FBN1 mRNA was shown to be ubiquitous across all human tissues and most prominent in adipose tissue throughout the body [Romere et al., 2016]. In line with these findings, the murine ortholog of FBN1 was shown to be expressed at high levels in white adipose tissue [Romere et al., 2016]. Genetic ablation of adipose tissue using Bscl2 ${ }^{-1-}$ mice (BSCL2 deficiency leads to 60$70 \%$ reduction in adipose tissue and is associated with Berardinelli-Seip congenital lipodystrophy) has proved adipose tissue as the main source of asprosin production [Romere et al., 2016].

\section{Asprosin in Clinical Conditions Associated with Impaired Glucose Levels}

It is not hard to predict that asprosin is involved in diseases associated with impaired glucose levels. Accumulating evidence suggests that serum asprosin concentrations are significantly enhanced in patients with type II diabetes (T2DM). In a study comprising 84 individuals with T2DM, serum asprosin concentrations were shown to be highly elevated in patients with T2DM compared to healthy control subjects, and high serum asprosin levels were identified to be important risk factors for T2DM pathogenesis [Zhang L et al., 2019]. In another study, individuals with impaired glucose levels and newly diagnosed T2DM patients were also shown to have increased plasma asprosin levels compared to subjects with normal glucose regulation, and plasma asprosin levels were reported to be positively correlated with the hemoglobin A 1c (HbA1c), fasting blood glucose (FBG), and triglyceride levels and insulin resistance [Wang et al., 2018]. Con- sistently, Li et al. [2018] reported that plasma asprosin levels were significantly elevated in female patients with T2DM in contrast to healthy subjects and were positively associated with $\mathrm{HbAlc}$, FBG, and insulin resistance. Zhang X et al. [2020] reported that circadian oscillation was disrupted in T2DM patients with elevated serum asprosin levels. Patients with normal glucose tolerance showed reduced postprandial asprosin levels compared to those with T2DM. Asprosin levels were negatively correlated with the blood glucose and C-peptide levels in subjects with normal glucose tolerance, but not in T2DM patients [Zhang X et al., 2020], suggesting that loss of asprosin response might be the main reason for fluctuating glucose levels in T2DM patients. T2DM patients with and without diabetic kidney disease were reported to have markedly higher circulating asprosin concentrations as compared to healthy individuals with normal glucose tolerance, and asprosin concentrations were positively correlated with the albumin-to-creatinine ratio but negatively correlated with the estimated glomerular filtration rate in T2DM patients [Zhang $\mathrm{H}$ et al., 2020]. These findings further supported the notion that asprosin might be responsible for the augmentation of the kidney damage in these patients. As a further supporting evidence, Naiemian et al. [2020] reported increased serum asprosin concentrations in T2DM patients compared to healthy subjects. Notably, asprosin was shown to be well correlated with FBG and body mass index (BMI) of healthy individuals and with $\mathrm{HbA1c}$, FBG, BMI, quantitative insulin check index, homeostatic model assessment of insulin resistance (HOMA-IR), total cholesterol/high-density lipoprotein cholesterol (TC/HDL-C) ratio, and triacylglycerol in patients with T2DM. Similarly, Gozel and Kilinc [2021] reported that T2DM patients have higher levels of asprosin compared to healthy subjects, and the circulating levels of asprosin were reduced following 3 months of metformin treatment in T2DM patients. Salivary asprosin concentrations were also shown to be high in these T2DM patients. Moreover, Groener et al. [2019] demonstrated that levels of plasma asprosin were not associated with the hypoglycemia unawareness and insulin resistance of type 1 diabetes mellitus patients.

Furthermore, greater levels of serum asprosin have been linked to albuminuria and renal function in T2DM patients. Serum asprosin levels of T2DM patients with microalbuminuria (albumin/creatinine ratio $\geq 30$ to $<300$ $\mathrm{mg} / \mathrm{g}$ ) and macroalbuminuria (albumin/creatinine ratio $\geq 300 \mathrm{mg} / \mathrm{g}$ ) were shown to be significantly higher than in T2DM patients with normoalbuminuria (albumin/creatinine ratio $<30 \mathrm{mg} / \mathrm{g}$ ), suggesting that asprosin might 
play a key role in the onset and progression of diabetic nephropathy [Deng et al., 2020]. Consistently, another study also confirmed that serum asprosin levels were positively correlated with the albumin-to-creatinine ratio and diabetic nephropathy progression in patients with T2DM [Wang et al., 2021].

Higher levels of asprosin were also reported in patients with metabolic syndrome. Hong et al. [2021] reported that patients with metabolic syndrome had higher serum asprosin levels, and there was a strong positive association between higher asprosin levels and BMI, waist circumference, fasting and 2-h fasting plasma glucose, fasting insulin, triglycerides, monocyte chemoattractant protein-1, interleukin-6, and HOMA-IR index. Monoclonal antibodies targeting asprosin reduced blood glucose levels and were associated with the loss of appetite and body weight in mouse models of metabolic syndrome [Mishra et al., 2021]. In contrast to these observations, Kocaman and Kuloğlu [2020] revealed in a streptozotocin-induced diabetes model that serum asprosin levels in diabetic rats were markedly lower than in the control group.

Pregnant women (at 18-20 weeks of gestation) with gestational diabetes mellitus were shown to have increased circulating and umbilical plasma asprosin levels, indicating that altered levels of asprosin are responsible for the fluctuating glucose levels of the mother, which eventually severely affects the growing fetus [Zhong et al., 2020]. Also, high levels of asprosin were well correlated with the increased glucose concentration in hyperglycemic menopausal women and well correlated with the risk factors of metabolic syndrome [Wiecek et al., 2019]. Also, asprosin levels were shown to be reduced with the wholebody cryotherapy in parallel to glucose levels in these patients [Wiecek et al., 2019].

Elevated levels of asprosin were also correlated with the insulin resistance of women with polycystic ovary syndrome (PCOS) compared to age- and BMI-matched healthy individuals and were associated with the BMI and free androgen index of these patients [Alan et al., 2019]. Similarly, plasma asprosin levels were shown to be increased in patients with PCOS but lower than in patients with T2DM, and a significant positive correlation was identified between high asprosin levels and FBG, insulin resistance, HbA1c, APOB, APOE, and LDL-c and testosterone levels [Li et al., 2018]. Likewise, Deniz et al. [2021] also reported increased asprosin levels in PCOS patients. In contrast to these observations, serum asprosin levels of PCOS patients were shown to be similar to those of control subjects in a different study population [Chang et al., 2019].

Asprosin in Health and Disease
Besides, elevated circulating serum asprosin levels were also implicated in the insulin resistance of non-alcoholic fatty liver disease patients and can be a novel promising biomarker for the differential diagnosis of non-alcoholic fatty liver disease [Ke et al., 2020].

Lastly, serum asprosin levels were reported to be dramatically reduced following coffee consumption in healthy individuals. Also, rapidly metabolizing individuals presented a higher reduction in blood asprosin concentrations compared to slow/intermediate metabolizers following caffeine consumption [Gkouskou et al., 2022].

\section{Asprosin, Appetite, Obesity, and Exercise}

Obesity and obesity-related complications are highly prevalent worldwide. Thus, adipokines like asprosin have gained special attention for the treatment of obesity and related health manifestations. Asprosin was identified to be an orexigenic hormone that stimulates appetite and promotes adiposity. Circulating asprosin was shown to cross the blood-brain barrier and lead to direct stimulation of orexigenic AgRP+ neurons and suppression of anorexigenic proopiomelanocortin-positive neurons [Duerrschmid et al., 2017].

Asprosin levels were shown to be significantly higher in obese patients (BMI $>35 \mathrm{~kg} / \mathrm{m}^{2}$ ) compared to nonobese individuals. Significant reduction of asprosin was observed following 6 months of post-bariatric surgery [Wang CY et al., 2019]. Asprosin was shown to be positively correlated with the BMI of individuals and correlated with the severity of obesity [Ugur and Aydin, 2019]. Serum asprosin concentrations were also shown to be elevated and associated with the insulin resistance in obese children compared to control subjects [Wang $\mathrm{M}$ et al., 2019]. Likewise, in another population, serum asprosin concentrations of obese children were also shown to be higher than those of normal-weight children [Sünnetçi Silistre and Hatipoğl, 2020]. In a monosodium glutamate-induced mouse model of obesity, asprosin levels were significantly lowered by cannabinoid receptor 1 (CB1R) inhibition [Ma et al., 2018]. At the molecular level, mRNA expression levels of asprosin were shown to be significantly elevated in retroperitoneal and subcutaneous adipose tissues of high-fat diet rats [Yang et al., 2020]. Unlike these observations, Long et al. [2019] reported that circulating asprosin concentrations were lower in children with obesity than in non-obese children. Additionally, patients with anorexia nervosa had higher plasma asprosin levels compared to control subjects [Hu et 
al., 2021]. Lastly, Liu et al. [2021] reported that serum asprosin levels were markedly higher in obese children, especially those with non-alcoholic fatty liver disease, and positively correlated with FBG, BMI, levels of tumor necrosis factor-alpha and alanine aminotransferase.

Moreover, emerging observations indicate that exercise has significant beneficial effects on asprosin levels. Higher levels of asprosin were shown to be dramatically reduced in obese patients after aerobic exercise [Ceylan et al., 2020]. Also, acute anaerobic exercise was shown to increase asprosin levels in women [Wiecek et al., 2018]. In a streptozotocin-induced T1DM rat model, aerobic exercise training was shown to significantly lower asprosin levels [Ko et al., 2019]. These findings further suggested that exercise might have a great impact on the regulation of serum asprosin levels.

\section{Cardiovascular Problems Associated with Asprosin Deregulation}

Asprosin was also implicated in the formation of cardiovascular problems. Acara et al. [2018] identified aspro$\sin$ as a reliable biomarker to predict the degree of coronary pathology in patients with unstable angina pectoris. Asprosin was also identified as a promising prognostic biomarker for dilated cardiomyopathy patients. Lower asprosin levels in these patients were shown to be associated with poor prognosis, and higher asprosin levels protected against hypoxia-induced cell death and improved mitochondrial respiration in cardiomyoblasts [Wen et al., 2020]. In addition, higher serum asprosin levels were also reported in patients with coronary artery disease (CAD) and were associated with the enhanced risk of developing CAD [Moradi et al., 2021].

\section{Possible Role of Asprosin in Cancer and Cellular Apoptosis}

Sustained and uncontrolled proliferation of cancer cells involves not only deregulation of proliferative signaling but also reprogramming of cellular energy metabolism, a phenomenon known as Warburg effect [Warburg et al., 1927]. Cancer cells reprogram their energy metabolism to a state called "aerobic glycolysis" in which they largely limit their energy metabolism to glycolysis even in the presence of oxygen [Hanahan and Weinberg, 2011]. Although limited, emerging evidence indicates that asprosin is deregulated in several types of human cancers. Asprosin was identified to be a promising predictive marker for the early diagnosis of pancreatic cancer [Nam et al., 2021]. Using immunohistochemical analysis, Kocaman and Artaş [2020] detected higher asprosin immunoreactivity in patients with malignant mesothelioma compared to reactive mesothelial hyperplasia. Also, cancer patients with anorexia were reported to have significantly lower fasting plasma asprosin concentrations. However, no significant difference was identified between cancer patients and non-cancer patients [Du et al., 2021]. In ovarian cancer, asprosin was found to be differentially expressed across all different histological subtypes [Kerslake et al., 2021]. Moreover, apoptosis is a natural defense system against neoplastic growth [Hanahan and Weinberg, 2011]. Accumulating evidence suggests that asprosin is involved in the modulation of the cellular apoptotic machinery. Particularly, asprosin was shown to interfere with the apoptosis of mesenchymal stromal cells via modulating the ERK1/2-SOD2 pathway [Zhang $\mathrm{Z}$ et al., 2019]. Consistent with these observations, increased asprosin levels reduced apoptotic death of cardiac microvascular endothelial cells [Chen et al., 2019]. Taken together, asprosin seems to have great impact in carcinogenesis, but limited literature on asprosin in cancer and apoptosis makes it difficult to comment more about the role of asprosin in cancer and apoptotic cell death, and more comprehensive studies are needed in the future.

\section{Asprosin and Inflammatory Response}

Asprosin was shown to be associated with the inflammation of pancreatic $\beta$-cells. Palmitate treatment of human primary islets containing $\beta$-cells and mouse insulinoma MIN6 cells increased the asprosin levels and nuclear factor-kappa B phosphorylation and the release of TNF and MCP-1 pro-inflammatory cytokines. Glucose-stimulated insulin secretion and cell viability was also shown to be impaired in these cells. Genetic ablation of asprosin with RNA interference reversed these effects. In contrast, recombinant asprosin treatment of human primary islets and MIN6 cells enhanced inflammatory response, apoptosis, and cellular dysfunction through upregulating toll-like receptor 4 (TLR4) and c-Jun N-terminal kinase (JNK) [Lee et al., 2019]. These observations strongly indicate that palmitate-induced asprosin expression from $\beta$-cells leads to $\beta$-cell dysfunction and inflammation through modulating TLR4/JNK signaling pathway, and targeting asprosin in T2DM patients might protect against $\beta$-cell dysfunction. Further evidence has support- 
ed these findings. Jung et al. [2019] reported that asprosin impairs insulin secretion through inducing the phosphorylation of protein kinase $\mathrm{C}-\delta(\mathrm{PKC} \delta)$, inositol-requiring enzyme 1 (IRE-1), and eukaryotic initiation factor 2 (eIF2 $\alpha$ ), and CHOP expression and inflammatory markers (phosphorylation of I $\mathrm{KB}$ and nuclear translocated nuclear factor- $\kappa \beta$, and interleukin- 6 expression). Collectively, these observations suggest that increased expression of asprosin might have great impact on the development of insulin sensitivity by activating inflammatory response.

\section{Asprosin in Fertility}

Asprosin was shown to enhance progressive motility of sperm and improves male fertility through binding its target receptor, OLFR734, and activating cAMP signaling circuitry to promote hepatic glucose secretion [Wei et al., 2019]. Asprosin treatment improved sperm progressive motility in old mice, suggesting asprosin as a potential treatment option to enhance male fertility [Wei et al., 2019]. Intracerebroventricular infusion of asprosin was shown to enhance GnRH mRNA and protein expression levels in the hypothalamus and serum FSH, LH, testosterone hormone levels and associate with the increased sperm density and progressive motility in rats [Keskin et al., 2021]. In addition, asprosin was also implicated in the regulation of ovarian follicular function by inducing $\mathrm{LH}-$ induced theca cell (TC) androstenedione production and suppressing IGF1-induced TC proliferation [Maylem et al., 2021]. Shortly, these observations indicate that precise regulation of asprosin is essential for the preservation of human reproductive health.

\section{Conclusions and Future Perspectives}

Current evidence suggests that asprosin is a key regulatory factor for preserving the homeostasis of energy metabolism. One of the main functions of asprosin is the regulation of glucose production and modulation of insulin secretion. Thus, it might serve as a promising diagnostic and therapeutic target in conditions associated with impaired glucose production and insulin secretion. Collectively, asprosin seems to have a wide range of regulatory functions in human physiology. However, various functions of asprosin in human health remain unexplored and warrant further investigation. Notably, asprosin might be responsible for the continuous supply of glucose during malignant transformation of cancer cells and have significant roles in the altered energy metabolism of cancer cells.

\section{Conflict of Interest Statement}

The authors have no conflicts of interest to declare.

\section{Funding Sources}

There is no financial support to report.

\section{Author Contributions}

M.A.O. and I.B.: Conceptualization, methodology, writing original draft, review and editing.

\section{References}

Acara AC, Bolatkale M, Kızıloğlu İ, İbișoğlu E, Can Ç. A novel biochemical marker for predicting the severity of ACS with unstable angina pectoris: Asprosin. Am J Emerg Med. 2018;36(8):1504-5.

Alan M, Gurlek B, Yilmaz A, Aksit M, Aslanipour B, Gulhan I, et al. Asprosin: a novel peptide hormone related to insulin resistance in women with polycystic ovary syndrome. Gynecol Endocrinol. 2019;35(3):220-3.

Brautbar A, LeMaire SA, Franco LM, Coselli JS, Milewicz DM, Belmont JW. FBN1 mutations in patients with descending thoracic aortic dissections. Am J Med Genet A. 2010;152A(2): 413-6.
Ceylan Hİ, Saygın Ö, Özel Türkcü Ü. Assessment of acute aerobic exercise in the morning versus evening on asprosin, spexin, lipocalin-2, and insulin level in overweight/obese versus normal weight adult men. Chronobiol Int. 2020;37(8):1252-68.

Chang CL, Huang SY, Hsu YC, Chin TH, Soong YK. The serum level of irisin, but not asprosin, is abnormal in polycystic ovary syndrome patients. Sci Rep. 2019;9(1):6447.

Chen S, Wang X, Qiu CM, Hou JN, Wei XY, Xiang CX, et al. [Study of the Role and Mechanism of Asprosin/Spartin Pathway in Cardiac Microvascular Endothelial Injury Induced by Diabete Mellitus]. Sichuan Da Xue Xue Bao Yi Xue Ban. 2019;50(6):827-34.
Deng X, Zhao L, Guo C, Yang L, Wang D, Li Y, et al. Higher Serum Asprosin Level is Associated with Urinary Albumin Excretion and Renal Function in Type 2 Diabetes. Diabetes Metab Syndr Obes. 2020;13:4341-51.

Deniz R, Yavuzkir S, Ugur K, Ustebay DU, Baykus Y, Ustebay S, et al. Subfatin and asprosin, two new metabolic players of polycystic ovary syndrome. J Obstet Gynaecol. 2021;41(2): 279-84.

Du C, Wang C, Guan X, Li J, Du X, Xu Z, et al. Asprosin is associated with anorexia and body fat mass in cancer patients. Support Care Cancer. 2021;29(3):1369-75. 
Duerrschmid C, He Y, Wang C, Li C, Bournat JC, Romere $\mathrm{C}$, et al. Asprosin is a centrally acting orexigenic hormone. Nat Med. 2017;23(12): 1444.

Gkouskou KG, Georgiopoulos G, Vlastos I, Lazou E, Chaniotis D, Papaioannou TG, et al. CYP1A2 polymorphisms modify the association of habitual coffee consumption with appetite, macronutrient intake, and body mass index: results from an observational cohort and a cross-over randomized study. Int J Obes. 2022;46(1):162-8.

Goldblatt J, Hyatt J, Edwards C, Walpole I. Further evidence for a marfanoid syndrome with neonatal progeroid features and severe generalized lipodystrophy due to frameshift mutations near the 3' end of the FBN1 gene. Am J Med Genet A. 2011;155A(4):717-20.

Gozel N, Kilinc F. Investigation of plasma aspro$\sin$ and saliva levels in newly diagnosed type 2 diabetes mellitus patients treated with metformin. Endokrynol Pol. 2021;72(1):37-43.

Graul-Neumann LM, Kienitz T, Robinson PN, Baasanjav S, Karow B, Gillessen-Kaesbach G, et al. Marfan syndrome with neonatal progeroid syndrome-like lipodystrophy associated with a novel frameshift mutation at the $3^{\prime}$ terminus of the FBN1-gene. Am J Med Genet A. 2010;152(11):2749-55.

Groener JB, Valkanou A, Kender Z, Pfeiffenberger J, Kihm L, Fleming T, et al. Asprosin response in hypoglycemia is not related to hypoglycemia unawareness but rather to insulin resistance in type 1 diabetes. PLoS One. 2019; 14(9):e0222771.

Hanahan D, Weinberg RA. Hallmarks of cancer: the next generation. Cell. 2011;144(5):64674.

Hong T, Li JY, Wang YD, Qi XY, Liao ZZ, Bhadel P, et al. High Serum Asprosin Levels Are Associated with Presence of Metabolic Syndrome. Int J Endocrinol. 2021;2021:6622129.

Horn D, Robinson PN. Progeroid facial features and lipodystrophy associated with a novel splice site mutation in the final intron of the FBN1 gene. Am J Med Genet A. 2011;155A(4): $721-4$.

Hu Y, Xu Y, Zheng Y, Kang Q, Lou Z, Liu Q, et al. Increased plasma asprosin levels in patients with drug-naive anorexia nervosa.Eat Weight Disord. 2021;26(1):313-21.

Jacquinet A, Verloes A, Callewaert B, Coremans C, Coucke P, De Paepe A, et al. Neonatal progeroid variant of Marfan syndrome with congenital lipodystrophy results from mutations at the $3^{\prime}$ end of FBN1 gene. Eur J Med Genet. 2014;57(5):230-4.

Jung TW, Kim HC, Kim HU, Park T, Park J, Kim $\mathrm{U}$, et al. Asprosin attenuates insulin signaling pathway through PKC $\delta$-activated ER stress and inflammation in skeletal muscle. J Cell Physiol. 2019;234(11):20888-99.

Ke F, Xue G, Jiang X, Li F, Lai X, Zhang M, et al. Combination of asprosin and adiponectin as a novel marker for diagnosing non-alcoholic fatty liver disease. Cytokine. 2020;134:155184.
Kerslake R, Hall M, Vagnarelli P, Jeyaneethi J, Randeva HS, Pados G, et al. A pancancer overview of FBN1, asprosin and its cognate receptor OR4M1 with detailed expression profiling in ovarian cancer. Oncol Lett. 2021; 22(3):650

Keskin T, Erden Y, Tekin S. Intracerebroventricular asprosin administration strongly stimulates hypothalamic-pituitary-testicular axis in rats. Mol Cell Endocrinol. 2021;538:111451.

Ko JR, Seo DY, Kim TN, Park SH, Kwak HB, Ko KS, et al. Aerobic Exercise Training Decreases Hepatic Asprosin in Diabetic Rats. J Clin Med. 2019;8(5):666.

Kocaman N, Artaş G. Can novel adipokines, asprosin and meteorin-like, be biomarkers for malignant mesothelioma? Biotech Histochem. 2020;95(3):171-5.

Kocaman N, Kuloğlu T. Expression of asprosin in rat hepatic, renal, heart, gastric, testicular and brain tissues and its changes in a streptozotocin-induced diabetes mellitus model. Tissue Cell. 2020;66:101397.

Lee T, Yun S, Jeong JH, Jung TW. Asprosin impairs insulin secretion in response to glucose and viability through TLR4/JNK-mediated inflammation. Mol Cell Endocrinol. 2019; 486:96-104.

Li E, Shan H, Chen L, Long A, Zhang Y, Liu Y, et al. OLFR734 mediates glucose metabolism as a receptor of asprosin. Cell Metab. 2019;30(2): 319-28.e8.

Li X, Liao M, Shen R, Zhang L, Hu H, Wu J, et al. Plasma asprosin levels are associated with glucose metabolism, lipid, and sex hormone profiles in females with metabolic-related diseases. Mediators Inflamm. 2018;2018: 7375294.

Liu LJ, Kang YR, Xiao YF. Increased asprosin is associated with non-alcoholic fatty liver disease in children with obesity. World J Pediatr. 2021;17(4):394-9.

Long W, Xie X, Du C, Zhao Y, Zhang C, Zhan D, et al. Decreased Circulating Levels of Asprosin in Obese Children. Horm Res Paediatr. 2019;91(4):271-7.

Ma H, Zhang G, Mou C, Fu X, Chen Y. Peripheral CB1 Receptor Neutral Antagonist, AM6545, Ameliorates Hypometabolic Obesity and Improves Adipokine Secretion in Monosodium Glutamate Induced Obese Mice. Front Pharmacol. 2018;9:156.

Maylem ERS, Spicer LJ, Batalha I, Schutz LF. Discovery of a possible role of asprosin in ovarian follicular function. J Mol Endocrinol. 2021; 66(1):35-44.

Mishra I, Duerrschmid C, Ku Z, He Y, Xie W, Silva ES, et al. Asprosin-neutralizing antibodies as a treatment for metabolic syndrome. Elife. 2021;10:e63784.

Moradi N, Fouani FZ, Vatannejad A, Bakhti Arani A, Shahrzad S, Fadaei R. Serum levels of Asprosin in patients diagnosed with coronary artery disease (CAD): a case-control study. Lipids Health Dis. 2021;20(1):88.
Naiemian S, Naeemipour M, Zarei M, Lari Najafi M, Gohari A, Behroozikhah MR, et al. Serum concentration of asprosin in new-onset type 2 diabetes. Diabetol Metab Syndr. 2020;12:65.

Nam H, Kang S, Park MS, Kang S, Kim HS, Mai V-H, et al. A Serum Marker for Early Pancreatic Cancer with a Possible Link to Diabetes. J Natl Cancer Inst. 2021; djab191.

O'Neill B, Simha V, Kotha V, Garg A. Body fat distribution and metabolic variables in patients with neonatal progeroid syndrome. Am J Med Genet A. 2007;143A(13):1421-30.

Robinson PN, Booms P, Katzke S, Ladewig M, Neumann L, Palz M, et al. Mutations of FBN1 and genotype-phenotype correlations in Marfan syndrome and related fibrillinopathies. Hum Mutat. 2002;20(3):153-61.

Romere C, Duerrschmid C, Bournat J, Constable $\mathrm{P}$, Jain M, Xia F, et al. Asprosin, a fasting-induced glucogenic protein hormone. Cell. 2016;165(3):566-79.

Sünnetçi Silistre E, Hatipoğl HU. Increased serum circulating asprosin levels in children with obesity. Pediatr Int. 2020;62(4):467-76.

Takenouchi T, Hida M, Sakamoto Y, Torii C, Kosaki R, Takahashi T, et al. Severe congenital lipodystrophy and a progeroid appearance: Mutation in the penultimate exon of FBN1 causing a recognizable phenotype. Am J Med Genet A. 2013;161A(12):3057-62.

Ugur K, Aydin S. Saliva and Blood Asprosin Hormone Concentration Associated with Obesity. Int J Endocrinol. 2019;2019:2521096.

Wang CY, Lin TA, Liu KH, Liao CH, Liu YY, Wu VC, et al. Serum asprosin levels and bariatric surgery outcomes in obese adults. Int $\mathrm{J}$ Obes (Lond). 2019;43(5):1019-25.

Wang M, Yin C, Wang L, Liu Y, Li H, Li M, et al. Serum Asprosin Concentrations Are Increased and Associated with Insulin Resistance in Children with Obesity. Ann Nutr Metab. 2019;75(4):205-12.

Wang R, Lin P, Sun H, Hu W. Increased serum asprosin is correlated with diabetic nephropathy. Diabetol Metab Syndr. 2021;13(1):51.

Wang Y, Qu H, Xiong X, Qiu Y, Liao Y, Chen Y, et al. Plasma asprosin concentrations are increased in individuals with glucose dysregulation and correlated with insulin resistance and first-phase insulin secretion. Mediators Inflamm. 2018;2018:9471583.

Warburg O, Wind F, Negelein E. The metabolism of tumors in the body. J Gen Physiol. 1927; 8(6):519-30.

Wei F, Long A, Wang Y. The Asprosin-OLFR734 hormonal signaling axis modulates male fertility. Cell Discov. 2019;5:55.

Wen MS, Wang CY, Yeh JK, Chen CC, Tsai ML, Ho MY, et al. The role of Asprosin in patients with dilated cardiomyopathy. BMC Cardiovasc Disord. 2020;20(1):402.

Wiecek M, Szymura J, Maciejczyk M, Kantorowicz M, Szygula Z. Acute Anaerobic Exercise Affects the Secretion of Asprosin, Irisin, and Other Cytokines - A Comparison Between Sexes. Front Physiol. 2018;9:1782. 
Wiecek M, Szymura J, Sproull J, Szygula Z. Decreased blood asprosin in hyperglycemic menopausal women as a result of whole-body cryotherapy regardless of metabolic syndrome. J Clin Med. 2019;8(9):1428.

Yang Z, Jiang J, Huang J, Zhao Y, Luo X, Song L. [Effect of high-fat diet and exercise on asprosin and CTRP6 expression in subcutaneous and retroperitoneal adipose tissues in rats during mid-gestation]. Nan Fang Yi Ke Da Xue Xue Bao. 2020;40(10):1406-14.
Zhang H, Hu W, Zhang G. Circulating asprosin levels are increased in patients with type $2 \mathrm{di}$ abetes and associated with early-stage diabetic kidney disease. Int Urol Nephrol. 2020; 52(8):1517-22.

Zhang L, Chen C, Zhou N, Fu Y, Cheng X. Circulating asprosin concentrations are increased in type 2 diabetes mellitus and independently associated with fasting glucose and triglyceride. Clin Chim Acta. 2019;489:183-8.

Zhang X, Jiang H, Ma X, Wu H. Increased serum level and impaired response to glucose fluctuation of asprosin is associated with type 2 diabetes mellitus. J Diabetes Investig. 2020; 11(2):349-55.
Zhang Z, Tan Y, Zhu L, Zhang B, Feng P, Gao E, et al. Asprosin improves the survival of mesenchymal stromal cells in myocardial infarction by inhibiting apoptosis via the activated ERK1/2-SOD2 pathway. Life Sci. 2019;231: 116554.

Zhong L, Long Y, Wang S, Lian R, Deng L, Ye Z, et al. Continuous elevation of plasma asprosin in pregnant women complicated with gestational diabetes mellitus: A nested case-control study. Placenta. 2020;93:17-22. 